\title{
Motional effects between on-center and off-center substitutional nitrogen in silicon
}

\author{
Kouichi Murakami, Hitoshi Kuribayashi, and Kohzoh Masuda \\ Institute of Materials Science, University of Tsukuba, Tsukuba, Ibaraki 305, Japan
}

(Received 24 March 1988)

\begin{abstract}
We have found for the first time that the hyperfine splitting of the ESR of off-center substitutional nitrogen in silicon increases with increasing the temperature above $\approx 150 \mathrm{~K}$. A model is proposed in which a hypothetical on-center substitutional $\mathbf{N}$ site exists in an adiabatic potentialenergy minimum higher than that of the off-center substitutional $\mathbf{N}$. Motional averaging and narrowing among these configurations of the $\mathbf{N}$ center occur between ESR hyperfine lines and account for the increase in the hyperfine splitting. The fractional $s$ character and localization of the unpaired electron at the on-center $\mathbf{N}$ seem to be significantly increased compared to those at the off-center $\mathbf{N}$.
\end{abstract}

Nitrogen can be introduced into off-center substitutional sites in silicon by $\mathrm{N}$-ion implantation and subsequent pulsed-laser annealing. ${ }^{1,2}$ The dynamics of pulsed-laser annealing have been studied by several time-resolved measurements. $^{3-5}$ It is thought that the great effectiveness of pulsed-laser annealing for introducing the off-center substitutional $\mathbf{N}$ is caused by both the extremely fast recrystallization and rapid cooling. ${ }^{3}$ Brower ${ }^{1}$ revealed through ESR measurements that off-center substitutional N (labeled SL5) is trigonally distorted with $C_{3 v}$ symmetry about the $\langle 111\rangle$ axis at temperatures below $100 \mathrm{~K}$ and has a deep level, similar to the $\mathbf{N}$ impurity in a type- $1 b$ diamond. ${ }^{6}$ The distortion of substitutional $\mathbf{N}$ in $\mathbf{S i}$ is attributed to a pseudo-Jahn-Teller effect by recent theoretical calculations. $^{7}$

In the present Rapid Communication, we report the strong temperature dependence observed for a ${ }^{14} \mathrm{~N}$ ESR hyperfine (hf) spectrum and the possibility of an on-center substitutional $\mathbf{N}$ state. The temperature dependence can be explained in terms of motional effects of the unpaired electron among the four equivalent off-center $\mathbf{N}$ distorted along the $\langle 111\rangle$ axis and hypothetical on-center $\mathrm{N}$ with $T_{d}$ symmetry.

$\mathbf{N}$ ions $\left(\mathrm{N}_{2}^{+}\right)$were implanted in Czochralski-grown Bdoped (100) $\mathrm{Si}$ with the resistivity of 30-50 $\Omega \mathrm{cm}$ and (111) $\mathrm{Si}$ with the resistivity of $\approx 120 \Omega \mathrm{cm}$ at an acceleration energy of $70 \mathrm{keV}$ and a dose of $2 \times 10^{14} / \mathrm{cm}^{2}$. The implanted samples were annealed with a $Q$-switched ruby laser at energy densities from 1.2 to $1.5 \mathrm{~J} / \mathrm{cm}^{2}$. The ESR measurements were made at temperatures ranging from 77 to $550 \mathrm{~K}$ with an $X$-band spectrometer. Offcenter substitutional $\mathbf{N}$ is known to be annealed out at temperatures higher than $575 \mathrm{~K}$ for the $\mathrm{Si}$ samples implanted with a dose of $2 \times 10^{14} / \mathrm{cm}^{2}$.

We observe for the first time that the hf splitting of the ESR spectrum for off-center substitutional $N\left({ }^{4} \mathrm{~N}\right.$ : nuclear spin $I=1$, natural abundance $99.63 \%$ ) increases without any change in the $g$ value with increasing temperature. Typical ESR spectra observed at various temperatures for $H \|\langle 100\rangle$ are shown in Fig. 1. The hf splitting $\Delta H_{\mathrm{hfs}}$ is plotted as a function of temperature in Fig. 2. $\Delta H_{\mathrm{hfs}}$ begins to increase at a temperature of $150 \mathrm{~K}$. The
${ }^{14} \mathrm{~N}$ hf spectra observed for $H \|\langle 110\rangle$ and $\langle 111\rangle$ exhibited the motional broadening in the linewidth at temperatures between 100 and $150 \mathrm{~K}$; above $150 \mathrm{~K}$, motional averaging and narrowing were observed, ${ }^{9}$ as reported in Ref. 1 . These motional effects come from the rapid reorientation of the $\langle 111\rangle$ distortion in off-center $N$. Therefore, we surmise that the increase in $\Delta H_{\mathrm{hfs}}$ is related to motional effects since $\Delta \boldsymbol{H}_{\mathrm{hfs}}$ begins to increase at the onset of the motional narrowing. For comparison we measured also the temperature dependence of the ESR spectrum of the Si-SL6 center $^{1,8}$ which has a larger $\langle 111\rangle$ distortion. The Si-SL6 center exhibits no changes in $\Delta H_{\mathrm{hfs}}$, as shown in Fig. 2, and no motional effects. These indicate that the change in $\Delta H_{\mathrm{hfs}}$ is probably not due to a simple thermal expansion or gradual reduction in the $\langle 111\rangle$ distortion, but is strongly related to motional averaging and narrowing of
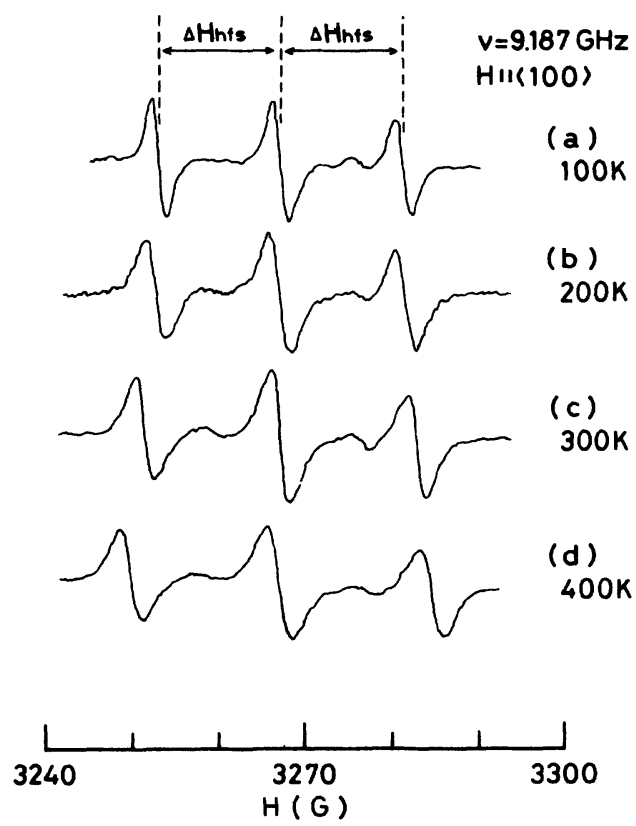

FIG. 1. Typical ESR spectra (a)-(d) of the substitutional ${ }^{14} \mathrm{~N}$ in $\mathrm{Si}$ measured at various temperatures. 


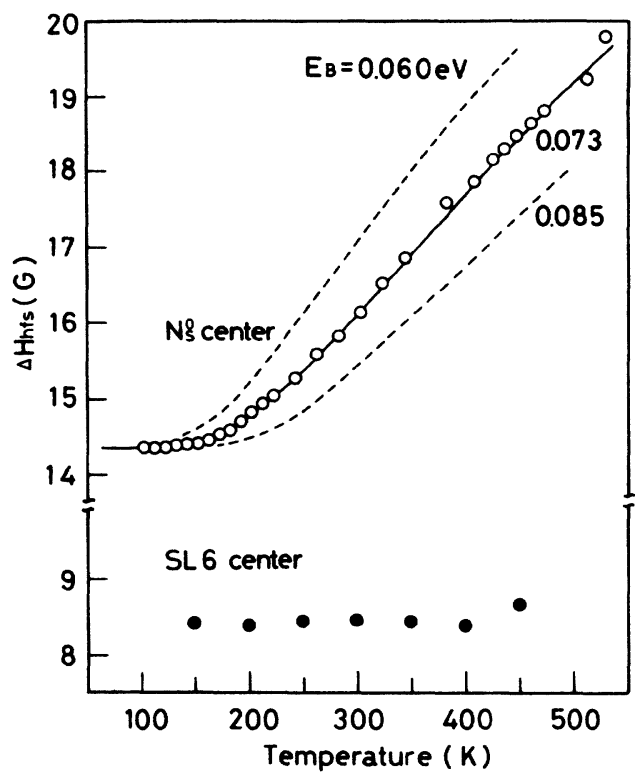

FIG. 2. Hyperfine splitting $\Delta H_{\mathrm{hfs}}$ of the ESR spectra of the substitutional $\mathbf{N}\left(\mathbf{N}_{s}^{0}\right)$ and SL6 center as functions of temperature. The solid curve corresponds to the best fitting values of $E_{B}=0.073 \mathrm{eV}$ and $\Delta H_{\mathrm{hfs}}\langle B\rangle=134 \mathrm{G}$, while dotted curves corresponding to other ones $(0.060 \mathrm{eV}$ and $134 \mathrm{G}$, and $0.085 \mathrm{eV}$ and $134 \mathrm{G}$ ) for $E_{B}$ are also shown for comparison.

the ESR spectrum which is caused by the rapid reorientation increasing with raising the temperature.

In order to explain the change in $\Delta H_{\mathrm{hfs}}$, we propose a model in which substitutional $\mathbf{N}$ has two types of minima in the adiabatic potential, i.e., a shallow on-center well and a deeper off-center well (in the $\langle 111\rangle$ direction), and that $\mathbf{N}$ goes through the lattice site in its reorientation from one $\langle 111\rangle$ distortion to another. The on-center minimum was predicted by theoretical calculations by Deleo, Fowler, and Watkins ${ }^{7}$ and Hjalmarson and Jennison. ${ }^{10}$ These states are schematically shown in Fig. 3, using a configuration coordinate potential-energy curve.

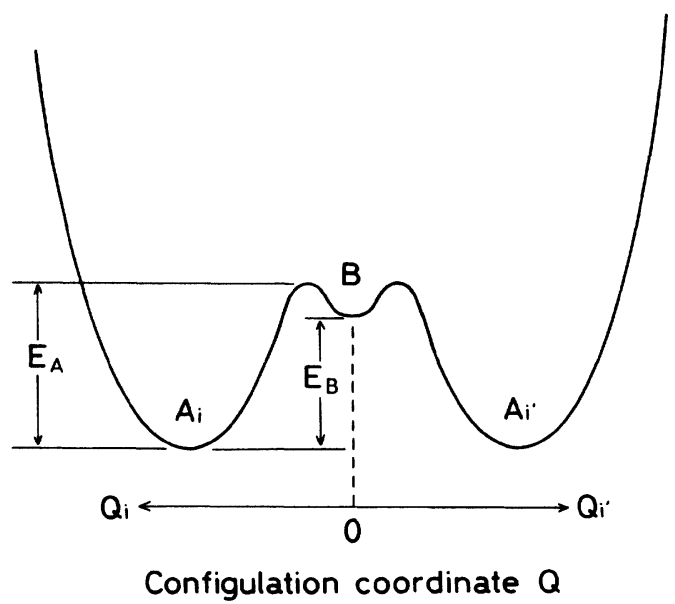

FIG. 3. Configuration coordinate potential-energy curve for the substitutional $\mathbf{N}$ in $\mathrm{Si}$. At the potential minimum $B$ at $Q_{i}=0, \mathrm{~N}$ occupies the substitutional lattice site, i.e., the oncenter site, and at the potential minima $A_{i}$ and $A_{i}{ }^{\prime}\left(i, i^{\prime}=1,2,3\right.$, or 4) $\mathbf{N}$ occupies the off-center site. For example, the configuration coordinate $Q_{i}$ and $Q_{i}$ correspond to the nitrogen

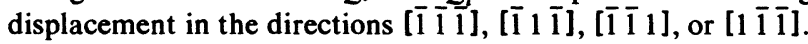

At temperatures higher than the onset $(150 \mathrm{~K})$ of motional averaging and narrowing, the unpaired electron moves around among states of the on-center minimum and the four equivalent off-center minima $A_{i}(i=1-4)$. We interpret our results in terms of the motional averaging and narrowing ${ }^{11,12}$ occurring between both the hf line for substitutional $\mathrm{N}$ in the off-center states $A_{i}$ and that for $\mathrm{N}$ in the on-center $B$ state which have the same $g$ value and different hf constants. In thermal equilibrium four $A_{i}$ states occur with equal population probability $p_{A}\left(\approx \frac{1}{4}\right)$ and the population probability $p_{B}$ at the on-center minimum $B$ becomes $\frac{1}{4} \exp \left(-E_{B} / k T\right)$ if $E_{B}>k T$. Here $E_{B}$ is the energy difference between the on-center minimum and off-center minima, as shown in Fig. 3. $\Delta H_{\mathrm{hfs}}(T)$ is represented by

$$
\Delta H_{\mathrm{hfs}}(T)=\Delta H_{\mathrm{hfs}}\langle A\rangle+\frac{1}{4} \exp \left(-E_{B} / k T\right)\left(\Delta H_{\mathrm{hfs}}\langle B\rangle-\Delta H_{\mathrm{hfs}}\langle A\rangle\right),
$$

where $\Delta H_{\mathrm{hfs}}\langle B\rangle$ and $\Delta H_{\mathrm{hfs}}\langle A\rangle$ are hf splittings of oncenter $\mathbf{N}$ and off-center $\mathbf{N}$, respectively.

As a result of fitting Eq. (1) to the experimental results, we obtain $E_{B}=0.073 \mathrm{eV}$ and $\Delta H_{\mathrm{hfs}}\langle B\rangle=134 \mathrm{G}$, as shown in Fig. 2. The value of the energy difference $E_{B}$ is compared to the barrier height $E_{A}=0.107 \mathrm{eV}$ (Fig. 3) for the reorientation among four equivalent off-center sites which was obtained by Brower. ${ }^{1}$ Furthermore, this result indicates that the on-center $\mathrm{N}$ has a deep level of $\approx 257 \mathrm{meV}$ $(=330-73)$, since the off-center $\mathbf{N}$ has a deep level of $\approx 330 \mathrm{meV} .{ }^{8,9}$ The wave functions of the unpaired electron for the on-center and off-center substitutional $\mathrm{N}$ are represented in terms of a one-electron antibonding molecular orbital,

$$
\psi\{i\}=\sum_{j} \eta_{j}\{i\}_{\phi_{j}}\{i\},
$$

where $i$ is either $A$ (off center) or $B$ (on center) and $\eta_{j}\{i\}^{2}\left(\Sigma_{j} \eta_{j}\{i\}^{2}=1\right)$ represents the degree of localization of the atomic orbital $\phi_{j}\{i\}$ at the $j$ th atomic site. The in$\operatorname{dex} j$ ranges over $\mathrm{N}$ and the surrounding $\mathrm{Si}$ atoms. Here,

$$
\phi_{j}\{i\}=\alpha_{j}\{i\}|s\rangle+\beta_{j}\{i\}|p\rangle,
$$

where $\alpha_{j}\{i\}$ and $\beta_{j}\{i\}\left(\alpha_{j}\{i\}^{2}+\beta_{j}\{i\}^{2}=1\right)$ are the fractions of the $s$ and $p$ character at the $j$ th atomic site, and $|s\rangle$ and $|p\rangle$ are $|2 s\rangle$ and $|2 p\rangle$ for the $\mathrm{N}$ atom and $|3 s\rangle$ and $|3 p\rangle$ for the $\mathrm{Si}$ atom, respectively. From the value obtained for $\Delta H_{\mathrm{hfs}}\langle B\rangle$, we estimate $\alpha_{\mathrm{N}}\{B\}^{2} \eta_{\mathrm{N}}\{B\}^{2}$ for the on-center substitutional $\mathrm{N}$ to be 0.241 , using a Fermicontact hf constant $A_{F}$ of $557 \mathrm{G}$ (Ref. 1) for the valence electron of a neutral $\mathbf{N}$ atom. The value is much larger than $\alpha_{N}\{A\}^{2} \eta_{\mathrm{N}}\{A\}^{2}$ of 0.0257 (Ref. 1) for off-center $\mathrm{N}$. This indicates that the $s$ character as well as the degree of 
localization at the on-center $\mathrm{N}$ atom are enhanced compared with the off-center $\mathbf{N}$. This result is consistent with an idea that the unpaired electron for the on-center $\mathbf{N}$ with $T_{d}$ symmetry occupies an $A_{1}$ singlet band-gap level. 7,13

If one of the four nearest $\mathrm{Si}$ sites surrounding $\mathrm{N}$ contains a ${ }^{29} \mathrm{Si}$ nucleus (nuclear spin $I=\frac{1}{2}$, natural abundance $4.7 \%$ ), then there is a hf interaction between the unpaired electron and the ${ }^{29} \mathrm{Si}$ nucleus. A study of the motional effects on the ${ }^{29} \mathrm{Si}$ hf satellites is also informative for testing our model. This is shown in Fig. 4 for $H \|\langle 100\rangle$. At temperatures lower than $130 \mathrm{~K}$ the ${ }^{29} \mathrm{Si} \mathrm{hf}$ satellites are clearly observed as in Figs. 4(a) and 4(b), while between 150 and $200 \mathrm{~K}$ the motional broadening occurs and so the intensity becomes very weak, as in Figs. 4(c) and 4(d). At temperatures above $200 \mathrm{~K}$ a motional averaged line that exhibited $\langle 111\rangle$ symmetry ${ }^{9}$ can be seen at a magnetic field of $\approx 3237 \mathrm{G}$ for $H \|\langle 100\rangle$ [Figs. 4(d)-4(f)]. This is just the averaged position between a ${ }^{14} \mathrm{~N}$ line $\left(I_{z}=+1\right)$ and a ${ }^{29} \mathrm{Si}$ line $\left(I_{z}=+\frac{1}{2}\right)$, indicated by two solid arrows in Fig. 4(a); the unpaired electron sees the ${ }^{29} \mathrm{Si}$ hf field with a probability of $\frac{1}{4}$. In the motionally averaged state, the ${ }^{29} \mathrm{Si}$ hf satellites were also found to be about four times as intense relative to the cen-

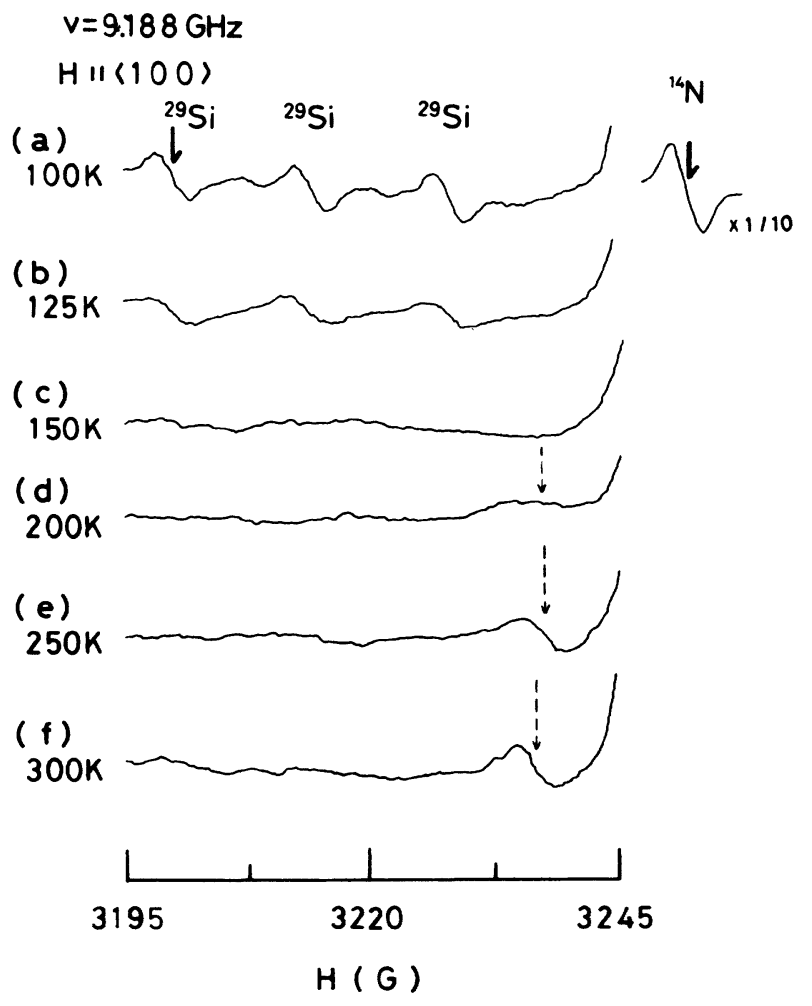

FIG. 4. Lower-field part of ${ }^{29} \mathrm{Si}$ hf lines in ESR spectrum of the substitutional $\mathrm{N}$ in $\mathrm{Si}$ for $H \|\langle 100\rangle$ and motional effects. Since the intensity of the ${ }^{29} \mathrm{Si}$ hf lines is very weak, we measured the ESR with a modulation-field of $5 \mathrm{G}$ larger than the ESR linewidth. The dotted arrows in (d)-(f) show the motionally averaged position between the lowest one of ${ }^{29} \mathrm{Si}$ hf satellites and the lowest one of ${ }^{14} \mathrm{~N}$ hf lines which are, respectively, indicated by the solid arrows in (a). tral ${ }^{14} \mathrm{~N}$ hf lines (see, e.g., Ref. 12).

Above $200 \mathrm{~K}$, we measured, moreover, the separation between the averaged ${ }^{29} \mathrm{Si}$ hf satellite and the low-field ${ }^{14} \mathrm{~N}$ hf line. The separation was found to decrease slightly with raising the temperature. We estimated from this result that $\alpha_{\mathrm{Si}}\{B\}^{2} \eta_{\mathrm{Si}}\{B\}^{2}$ was less than 0.02. ${ }^{9}$ The values $a^{2} \eta^{2}, \alpha^{2}$, and $\eta^{2}$ obtained for on-center $\mathrm{N}$ are given in Table I and are compared with those for off-center N. ${ }^{1}$ This result suggests that the degree of localization, $\eta_{\mathrm{S} i}\{B\}^{2}$, decreases down to $\$ 0.08$ if we make a guess of $25 \% s$ and $75 \% p$ for the atomic function of four equivalent $\mathrm{Si}$ atoms surrounding the on-center $\mathrm{N}$ atom. This result indicates that the unpaired electron density is $\$ 32 \%(=8 \times 4)$ localized on the nearest four Si sites. Thus the ${ }^{29} \mathrm{Si}$ hf structure indicates that even the oncenter $\mathbf{N}$ still gives strong nearest ${ }^{29} \mathrm{Si}$ neighbor interactions as observed for the deep group-VI donors, ${ }^{14}$ in contrast with the shallow group-V donors ( $\mathrm{P}, \mathrm{As}$, and $\mathrm{Sb}$ ) whose wave functions have nodes at the nearest neighbors. This also suggests that on-center $\mathbf{N}$ has a deep level rather than a shallow one.

Finally, we discuss the ratio of the $s$ and $p$ character and the degree of localization for the hypothetical oncenter $\mathbf{N}$ in $\mathrm{Si}$. According to a simple argument presented by Bachelet, Baraff, and Schlüter, ${ }^{13}$ the state of the unpaired electron for on-center $\mathrm{N}$ is an antibonding $A_{1}$ type consisting of the dangling-bond-like singlet $a_{1}$ state of the $\mathrm{Si}$ lattice vacancy and $2 s$ state of the $\mathrm{N}$ atom. Since the $2 s$ level of the $\mathrm{N}$ atom is further from the $A_{1}$ level than the $a_{1}$ energy, i.e., the hybridization is asymmetric, $\eta_{\mathrm{N}}\{B\}^{2}$ would be smaller than 0.5 . If we take $\eta_{\mathrm{N}}\{B\}^{2}$ to be 0.5 at most, then $\alpha_{\mathrm{N}}\{B\}^{2}$ becomes 0.48 , whereas if we take $\alpha_{N}\{B\}^{2}$ to be 1.0 (pure $s$-like) as in Refs. 7 and 13, $\eta_{\mathrm{N}}\{B\}^{2}$ is estimated to be 0.24 . These values estimated for the on-center $\mathbf{N}$ are much larger than those for the off-center $\mathrm{N}$, as summarized in Table I. The $82(=50+4$ $\times 8) \%-56(=24+4 \times 8) \%$ unpaired electron density for the wave function $\psi\{B\}$ is localized on on-center $\mathrm{N}$ and the surrounding four $\mathrm{Si}$ sites, while the remaining $18 \%-44 \%$ of $\psi\{B\}$ is presumably spread over more distant Si neighbors. This value for the degree of localization of $\psi\{B\}$ is comparable to that calculated for on-center $N$ in diamond ${ }^{13}$ and silicon. ${ }^{7}$ On the other hand, the remaining $18 \%$ of the wave function $\psi\{A\}$ is spread over the more distant Si neighbors. ${ }^{1}$ Thus, this localization and $s$-like character are not inconsistent with our configuration coordinate model in Fig. 3.

TABLE I. $s$ charactor and localization degree of the unpaired electron of substitutional $\mathbf{N}$ in $\mathrm{Si}$. The second column contains the values obtained by Brower (Ref. 1).

\begin{tabular}{ccc}
\hline \hline & On-center N $\{B\}$ & Off-center $N\left\{A_{i}\right\}(i=1-4)$ \\
\hline$\alpha{ }_{N}^{2} \eta_{N}^{2}$ & 0.241 & 0.0257 \\
$\alpha N_{N}^{2}$ & $1.0-0.48$ & 0.28 \\
$\eta_{N}^{2}$ & $0.24-0.5$ & 0.09 \\
$\alpha_{S i}^{2} \eta_{S i}^{2}$ & $\lesssim 0.02$ & 0.0876 \\
$\alpha_{S i}^{2}$ & $\approx 0.25$ & 0.12 \\
$\eta_{S i}^{2}$ & $\lesssim 0.08$ & 0.73 \\
\hline \hline
\end{tabular}


In conclusion, we have found for the first time that the hyperfine splitting of a ${ }^{14} \mathrm{~N}$ ESR sprectrum increases with increasing temperature above $\approx 150 \mathrm{~K}$. From this result, we have deduced that a shallow potential well at the $T_{d}$ substitutional site does exist, but has a configurational energy higher than that of the $\langle 111\rangle$ distorted configuration by approximately $0.073 \mathrm{eV}$. Our results demonstrate that ESR measurements at relatively high temperatures can also be informative with regard to excited defect configurations which may be important in understanding the more complete dynamics of point defects in semiconductors.

The authors would like to thank Professor H. Sumi for helpful discussion and Dr. H. Itoh (Japan Atomic Energy Research Institute, Takasaki Research Establishment) for his help in the initial stages of this work. This work was partly supported by a Grant-in-Aid for Scientific Research (1987-1988) from the Ministry of Education, Science, and Culture, Japan.

\section{9, 3193 (1984).}

${ }^{8}$ H. Itoh, K. Murakami, K. Takita, and K. Masuda, Mater. Sci. Forum 10-12, 899 (1986); J. Appl. Phys. 61, 4862 (1987).

${ }^{9}$ K. Murakami, H. Kuribayashi, and K. Masuda (unpublished).

${ }^{10}$ H. P. Hjalmarson and D. R. Jennison, Phys. Rev. B 31, 1208 (1985).

${ }^{11}$ P. W. Anderson, J. Phys. Soc. Jpn. 9, 316 (1954).

${ }^{12}$ G. D. Watkins and J. W. Corbett, Phys. Rev. 134, A1359 (1964).

${ }^{13}$ G. B. Bachelet, G. B. Baraff, and M. Schlüter, Phys. Rev. B 24, 4736 (1981).

${ }^{14}$ H. G. Grimmeiss, E. Janzén, H. Ennen, O. Schirmer, J. Schneider, R. Wörner, C. Holm, E. Sirtle, and P. Wagner, Phys. Rev. B 24, 4571 (1981). 